Doi: $10.4274 /$ jus.2017.04.025

\title{
Re: Physical Aspects of Cancer Invasion
}

\author{
Guiot C, Pugno N, Delsanto PP, Deisboeck TS
}

University of Torino, Department of Neuroscience, CNISM, Torino, Italy

Phys Biol 2007;4:P1-6. doi: 10.1088/1478-3975/4/4/P01.

\section{EDITORIAL COMMENT}

One of the current research topics is invasion mechanism of cancer cells. Invasiveness, one of the properties of tumor progression, contains the tumor's ability to expand into the host tissue due to biochemical and biomechanical processes. Invasion mechanisms are investigated on models ranging from microscopic to macroscopic scales. Microscopic models are suitable to describe malignant transformations in the cellular level (biochemical, genetic, etc.). Macroscopic models of solid tumor growth may be related to the universal laws of physics (pressure, heat, etc.). Another described model is mesoscopic version and this design is intermediate type between microscopic and macroscopic levels. Tumor invasion is controlled by three parameters: tumor surface tension, microenvironmental pressure and tumor radius. The authors noted the two main cancer invasive mechanisms, namely "smooth margin" invasive mass and "fingering", as a progressive damage growth in comminuted solids or a drop splashing in liquids.

They suggested that "composite" models are more logical to explain cancer invasion mechanism. Additionally, for diagnostic and therapeutic purposes of the neoplastic growth, a multilevel approach that includes both micro and macroscopic scales and its mesoscopic bridging level may be most promising. For completely understanding the invasion mechanisms of the cancer cells, there is a need for further research about this topic.

Fehmi Narter, MD, PhD

๑Copyright 2017 by the Association of Urological Surgery / Journal of Urological Surgery published by Galenos Publishing House. 